\title{
Author Correction: Post-traumatic stress disorder affects fucose-a(1-2)-glycans in the human brain: preliminary findings of neuro deregulation using in vivo two- dimensional neuro MR spectroscopy
}

Scott Quadrelli ${ }^{1,2,3,4}$, Nathan Tosh ${ }^{1,3}$, Aaron Urquhart ${ }^{1}$, Katie Trickey ${ }^{1}$, Rosanna Tremewan ${ }^{1}$, Graham Galloway (D) , Lisa Rich ${ }^{1}$, Rodney Lea ${ }^{3}$, Peter Malycha ${ }^{1}$ and Carolyn Mountford ${ }^{1,2}$

\section{Correction to: Translational Psychiatry; https://doi.org/10.1038/s41398-018-0365-6; Published online 18 January 2019}

The original article contained errors in the Fig. 1 caption. The incorrect sentence, "The region highlighted by the white box is expanded in Fig. 3" was corrected to, "The region highlighted by the white box is expanded in Fig. 2." This has been corrected in the HTML and PDF of the article.

Published online: 05 February 2019 